\title{
Uso do GQM para Avaliar Documentos de Utilização de Framework
}

\author{
Fernando Mauro de Souza ${ }^{1}$, Adílson Marques da Cunha ${ }^{1}$, Clovis Torres \\ Fernandes $^{1}$, Eduardo Martins Guerra ${ }^{1}$ \\ ${ }^{1}$ Divisão de Pós-Graduação - Instituto Tecnológico de Aeronáutica (ITA) \\ Praça Marechal Eduardo Gomes, 50 - Vila das Acácias \\ CEP: 12228-900 - São José dos Campos, SP - Brasil \\ fermauros@gmail.com, adicunha@directnet.com.br, clovistf@uol.com.br, \\ guerraem@gmail.com
}

\begin{abstract}
Many approaches to document frameworks have been suggested in the literature of framework reuse, some show how effective they may be in reducing the learning curve of using the former. However, there is still a lack of research to measure these documents quality. This article describes an approach of framework documentation denominated Guide Cooperation Framework (GCF) and a model of quality and issues, based on the Goal /Question/Metrics (GQM) model, whose aim is to help the interpretation of the quality measuring results of the framework documentation. The proposed model assesses the documentation ease of understanding and its usability.
\end{abstract}

Resumo. Muitas abordagens para documentar frameworks têm sido sugeridas na literatura para o reúso de framework, algumas mostram como podem ser efetivas na redução da curva de aprendizagem de utilização do mesmo. Entretanto, ainda há uma carência de trabalhos de investigação para medir a qualidade destes documentos. Neste artigo é apresentada uma abordagem de documentação de framework denominada Guia de Colaboração de Framework (GCF) e um modelo de qualidade e questões, baseadas no modelo Goal/Question/Metrics (GQM), com o objetivo de auxiliar na interpretação dos resultados da medição da qualidade desses. $O$ modelo aqui proposto avalia a facilidade de entendimento da documentação e a sua usabilidade.

\section{Introdução}

Frameworks orientados a objetos são técnicas poderosas de reutilização de software capazes de oferecer níveis elevados de projeto e reúso de código. Os frameworks permitem uma produtividade mais elevada do desenvolvimento, um menor tempo de produção, e uma maior qualidade das aplicações. No entanto, estes benefícios são adquiridos ao longo do tempo e requerem dedicação e esforço considerável na aprendizagem e compreensão dos detalhes essenciais da arquitetura do projeto e de como utilizá-lo. Uma boa documentação é essencial para o sucesso do mesmo. Sem uma documentação clara, completa e precisa descrevendo como se utilizar o framework, como está projetado e a forma de se trabalhar com ele, será muito difícil para entendê-lo e utilizá-lo. Muitas abordagens de documentação têm sido sugeridas na literatura e algumas mostram como podem ser efetivas na redução da curva do tempo de aprendizagem de utilização, tais como Livro de Receitas (Cookbook) [Johnson 1992], Linguagem padrão [Krasner 1988], e Meta padrão [Pree 1995]. Grady Booch [1994] 
afirma que "A maior parte dos frameworks nunca será reutilizada a menos que o custo de entendê-lo e então utilizar suas abstrações seja menor que o custo de compreensão dos programadores em escrevê-las do zero". Neste artigo apresentamos um modelo de métricas, que foi definido utilizando o Goal/Question/Metric [Basili 1994] para avaliar e, assim, apontar alguns resultados de uma nova abordagem de documentação de framework denominada GCF [Souza 2008]. Na seção 2 será apresentada a abordagem de documentação denominada GCF, na seção 3 serão apresentados os conceitos referentes a métricas de software. Na seção 4 será apresentado o modelo de métricas para avaliação do GCF através da aplicação da abordagem GQM. Na seção 5 serão definidas as métricas, coleta e interpretação dos resultados obtidos através da aplicação prática dessas métricas. Finalmente, na seção 6 serão apresentadas as conclusões deste trabalho.

\section{O Guia de Colaborações de Framework (GCF)}

O GCF é uma abordagem que inclui informação que explica como usar o framework sob diferentes perspectivas (vistas externa, interna, estática e dinâmica), em diferentes níveis de abstração e detalhe (arquitetura, imagem, código), e para tal agrega características de diversos tipos de documentos (Figura 1). Para habilitar os usuários a utilizar o framework, a documentação combina informações prescritivas, exemplos concretos, e ajuda o usuário a aprender e experimentar (Figura 2) [Souza 2008]. As informações descritivas relacionadas (padrões, contratos, etc.) estão disponíveis, e a abordagem explica como trabalhar com o framework, além de mostrar os detalhes concretos das implementações para os leitores que procuram um melhor entendimento interno do framework. A documentação é escrita pelo ponto de vista do leitor, ordenada de modo que reflita uma liberdade de uso, com explicação das informações mais importantes que devem ser apresentadas.

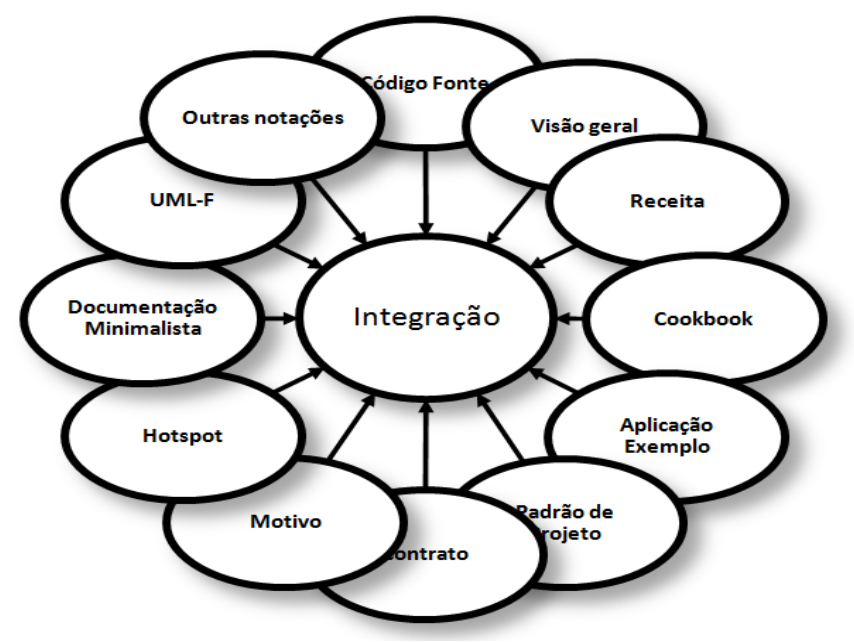

Figura 1. Conjunto das Técnicas de Documentação [Souza 2008]

Assim, o GCF aborda diversos requisitos, entre os quais se destacam os seguintes:

- Facilidade de uso pelos programadores, para que a atividade de documentação não constitua um obstáculo à atividade de programação;

- Flexibilidade para se adaptar às particularidades de cada ambiente de projeto;

- Capacidade de interligar os diferentes tipos de conteúdos produzidos; 


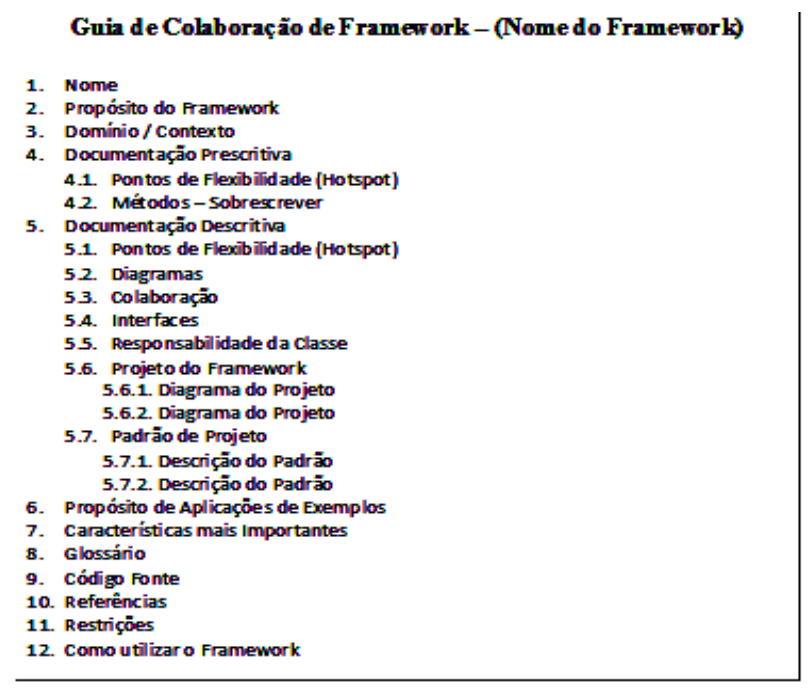

Figura 2. Estrutura do GCF [Souza 2008]

\section{Métricas de software}

Segundo Fenton e Pfleeger [1994] medição de software é o processo através do quais números e símbolos são atribuídos ao mundo real de forma a tornar possível caracterizar cada entidade através de regras claramente definidas. A medição se dá através da aplicação de métricas de processos, produtos e serviços. Uma métrica pode ser definida, por sua vez, com uma "definição matemática, algorítmica ou função usada para obter uma avaliação quantitativa de um produto ou processo". Segundo a norma ISO (International Organization for Standardization) [2001], métrica é a composição de métodos para medição e escalas de medição. Essas escalas de medição são formas de mapeamento que permitem entender o comportamento das entidades por meio da manipulação de dados. Existem diferentes tipos de escalas [Fenton 1994]:

- Nominal: provê um nome ou um valor para um atributo, no entanto, a ordem dos valores não tem nenhum significado para a sua interpretação.

- Ordinal: os resultados estão em uma determinada ordem (ascendente ou descendente), mas a distância entre os pontos dessa escala não tem significado.

- Intervalo: preserva a importância da ordem dos resultados, e possui informações sobre o tamanho dos intervalos que separaram os pontos da escala.

- Racional: semelhante à escala de intervalo, no entanto ela representa também as proporções entre as entidades, possui o marco zero (indicador da falta do atributo).

- Absoluta: feita através da contagem do número de elementos de uma entidade.

Para que a medição seja efetiva, torna-se necessário focá-la nos objetivos a serem alcançados com tais medições [Basili 1994]. Pfleeger [1994] destaca que é necessário deixar claras as necessidades para poder melhor conhecer os objetivos das medições. O uso de modelos pode guiar a definição de aplicação de medições a serem realizadas, um modelo comumente utilizado é o GQM [Basili 1994] [SAGP 2008].

\section{A metodologia GQM (Goal/Question/Metrics)}

A abordagem GQM (Figura 3) se apresenta como um mecanismo para planejamento, definição de metas de medição e avaliação. O objetivo do método GQM é caracterizar e fornecer um melhor entendimento dos processos, produtos, recursos e ambientes e, 
assim, estabelecer bases para comparação com trabalhos futuros [Soligen 1999]. O GQM baseia-se na suposição de que para se medir de maneira eficaz, alguns objetivos devem ser estabelecidos para que estes sirvam de rota para o estabelecimento de questões que orientarão a definição de métricas em um contexto particular. É muito importante para o sucesso da aplicação do GQM que os objetivos estejam bem traçados, pois somente assim a escolha das métricas e posterior avaliação dos dados será bem sucedida [Gomes 2001]. Sendo assim o GQM considera um modelo com três níveis de realização:

- Conceitual - Definição do escopo da avaliação, ou seja, do objeto a ser medido. (Processos, Produtos ou Recursos)

- Operacional - Definição de um conjunto de questões que auxilie na caracterização do objeto de estudo e como ele deve ser enxergada dentro do contexto da qualidade.

- Quantitativo - Definição de um conjunto de dados a serem obtidos, relacionado a cada uma das questões definidas anteriormente, a fim de respondê-las de forma quantitativa, ou seja, as métricas.

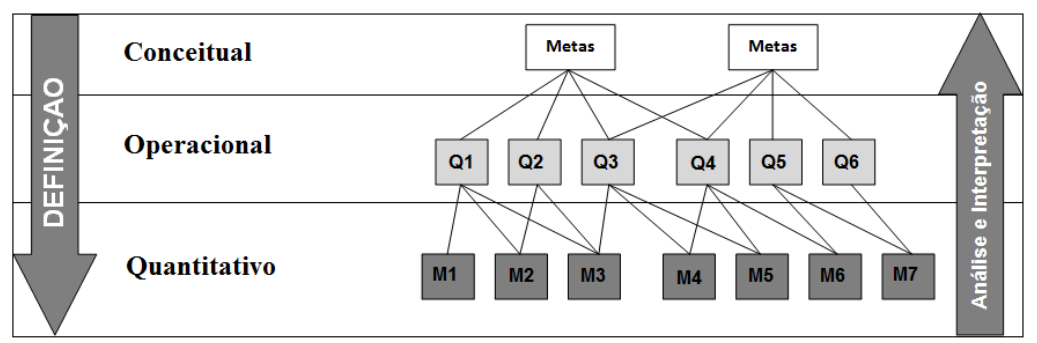

Figura 3. Metodologia GQM [Soligen 1999].

A Figura 4 apresenta a forma como Solingen e Berghout [1999] definem as fases do método GQM.

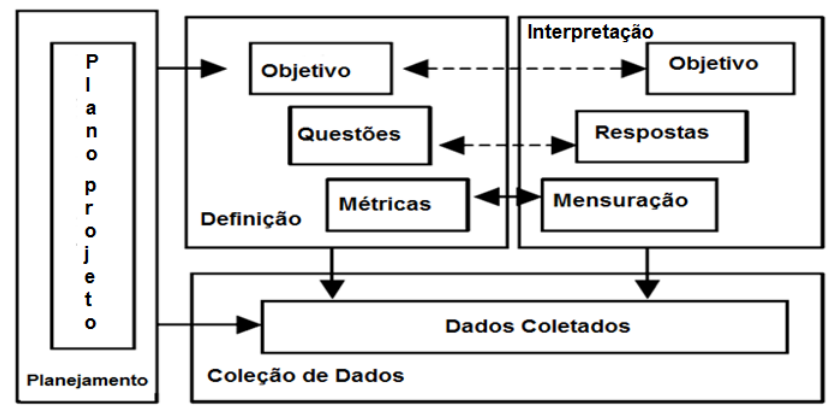

Figura 4. Fases do método GQM [SAGP 2008]

- Planejamento - nesta fase serão realizadas as seguintes atividades: relacionar a equipe que participará do GQM, selecionar a área que se deseja melhorar, apontar os projetos que farão parte da aplicação do método e treinamento da equipe nos conceitos necessários para a aplicação do GQM.

- Definição - nesta fase dever-se-á definir os objetivos do GQM, produzir ou adaptar modelos de software, definir as questões a serem respondidas, definir e refinar as métricas, além de promover a revisão dos planos do GQM.

- Coleta de Dados - Os dados são coletados, com base nas métricas definidas.

- Interpretação - Os dados coletados anteriormente são absorvidos e conclusões acerca dos mesmos são extraídas pela equipe de GQM. Com base neles as questões definidas podem ser respondidas. 


\section{Definição de métricas para avaliação da documentação de framework}

Para aplicar o GQM na definição de métricas para avaliação da documentação seguimos o conjunto de fases apresentadas anteriormente. Na fase de planejamento a equipe é formada por alunos do $5^{\circ}$ ano de graduação do curso de engenharia eletrônica e computação do Instituto Tecnológico de Aeronáutica - ITA, matéria CES-26 (Tópicos Avançados em Orientação a Objetos). Na fase de definição identificamos dois objetivos de medição: avaliar a facilidade de entendimento da documentação de utilização de framework [Ramos 2004] e a usabilidade [Usability 2008] da estrutura de documentação de framework proposta pelos alunos no trabalho de fim de curso em relação ao GCF. Para cada objetivo definimos questões de avaliação e métricas para avaliá-las. A seguir são apresentados os dois objetivos de medição, assim como suas questões e métricas correspondentes.

\subsection{Avaliação da documentação de utilização de framework}

O primeiro objetivo de medição foi definido para avaliar a documentação de utilização de framework. A Tabela 1 apresenta a formalização desse objetivo segundo a estrutura proposta por [Soligen 1999].

Tabela 1. Objetivo de medição 1

\begin{tabular}{|l|l|}
\hline Analisar a $\quad-$ & documentação de utilização de framework \\
\hline Com o propósito de - & avaliar a facilidade de entendimento da documentação de utilização de framework [Ramos 2004] \\
\hline Com respeito a - & completude e facilidade de entendimento \\
\hline Do ponto de vista - & desenvolvedor de aplicações \\
\hline No contexto do - & desenvolvimento de aplicações \\
\hline
\end{tabular}

Duas questões foram derivadas para atender esse objetivo: Questão 1.1: Qual o grau de compreensão da linguagem utilizada pela documentação do framework? Questão 1.2: Qual o grau de compreensão das ilustrações e exemplos utilizados na documentação? Para definir as métricas que respondessem essas questões consideramos então avaliar: as características gerais da documentação (Nome do framework, Propósito, Domínio, Contexto), itens da Documentação prescritiva (Pontos de Flexibilidade - Hotspot), itens da Documentação Descritiva (Diagramas, Padrões de Projeto), características específicas (Exemplos, Características mais importantes, glossário, Código Fonte, Referências, Restrições) e Como utilizar o framework [Souza 2008]. Algumas métricas foram definidas a partir da experiência do autor e outras foram selecionadas da literatura [Usability 2008] utilizando-se um tipo de escala específico definidas na seção anterior. Para auxiliar na interpretação das medições, subjetivas, coletadas para as métricas foi proposto quando necessário uma escala ordinal que permita a melhor avaliação [Ramos 2004]. A tabela 2 representa a escala de avaliação das métricas. As Tabelas 3 e 4 apresentam as métricas definidas para as questões 1 e 2 respectivamente.

Tabela 2. Escala de avaliação das métricas

\begin{tabular}{|c|l|l|l|l|l|}
\hline Fórmula & \multicolumn{5}{|c|}{ Interpretação } \\
\hline $\mathrm{X}=\mathrm{A}$, Onde: $\mathrm{A}=$ & (5) Muito Satisfeito & (4) Média & (3) Satisfeito & (2) Pouco Satisfeito & (1) Insatisfeito \\
\hline
\end{tabular}

Tabela 3. Métricas para Objetivo 1 - Questão 1

Questão 1.1: Qual o grau de compreensão da linguagem utilizada pela documentação de framework?

M1.1.1) Grau de facilidade para compreender e completar as tarefas de forma rápida e eficiente com o auxílio da documentação?

M1.1.2) Grau de adequação do conteúdo da documentação? 


\section{Tabela 4. Métricas para Objetivo 1 - Questão 2}

Questão 1.2: Qual o grau de compreensão das ilustrações e exemplos utilizados na documentação?

M1.2.1) Grau de facilidade para compreender as ilustrações e exemplos utilizados e completar as tarefas de forma rápida e eficiente?

M1.2.2) Grau de adequação das ilustrações e exemplos utilizados no conteúdo da documentação ?

\subsection{Avaliação da usabilidade da documentação de framework}

O segundo objetivo de medição definido é usado para avaliar a usabilidade da documentação de framework. A Tabela 5 apresenta a formalização desse objetivo segundo a estrutura proposta por [Soligen 1999].

\section{Tabela 5. Objetivo de medição 2}

\begin{tabular}{|ll|l|}
\hline Analisar a $\quad-$ & usabilidade da documentação do framework \\
\hline Com o propósito de - & avaliar a usabilidade da documentação [Usability 2008] \\
\hline Com respeito a - & capacidade que a documentação tem de ser entendida, aprendida, utilizada e atraente para o usuário \\
\hline Do ponto de vista - & desenvolvedor de aplicações \\
\hline No contexto do - & desenvolvimento de aplicações \\
\hline
\end{tabular}

Duas questões foram derivadas para atender esse objetivo: Questão 2.1: Qual o grau do nível de detalhe da documentação de utilização de framework? Questão 2.2: Qual o grau de satisfação do usuário após utilizarem a documentação de framework? Para definir as métricas que respondessem essas questões, consideramos então avaliar: a capacidade da documentação ser inteligível e colaborar para que o usuário possa entendê-lo. As Tabelas 6 e 7 apresentam as métricas definidas para as questões 1 e 2 respectivamente.

\section{Tabela 6. Métricas para Objetivo 2 - Questão 1}

\begin{tabular}{|l|}
\hline Questão 2.1: Qual o grau do nível de detalhe da documentação de utilização do framework? \\
\hline M2.1.1) Grau de satisfação com a apresentação da documentação ? \\
\hline M2.1.2) Grau de integração entre os assuntos da documentação? \\
\hline M2.1.3) Grau de clareza da documentação ? \\
\hline
\end{tabular}

Tabela 7. Métricas para Objetivo 2 - Questão 2

\begin{tabular}{|l|}
\hline Questão 2.2: Qual o grau de satisfação do usuário após utilizarem a documentação de framework? \\
\hline M2.2.1) Grau do nível de satisfação da leitura e simplicidade da documentação ? \\
\hline M2.2.2) Grau de consistência da documentação \\
\hline M2.2.3) Grau de facilidade de manipulação da documentação \\
\hline
\end{tabular}

\subsection{Coleta de Dados}

Para a coleta de dados foram definidas dez métricas, inicialmente descritas nas Tabelas 3, 4, 6 e 7. Na fase da coleta de dados foi realizada através de questionário distribuído em duas etapas, sendo elas no quinto e nono período, aos alunos do $5^{\circ}$ ano de graduação do curso de engenharia eletrônica e computação do Instituto Tecnológico de Aeronáutica - ITA, matéria CES-26 (Tópicos Avançados em Orientação a Objetos).

\subsection{Interpretação}

A etapa de interpretação da coleta de dados ocorreu ao final do semestre do curso de graduação do ITA. Esta etapa foi conduzida pelo autor deste artigo, onde o mesmo tem a consciência de que não bastou apenas a definição de uma estrutura padronizada, mas também que a mesma esteja associada a conteúdos melhores elaborados pelos desenvolvedores de documentação de framework. Assim sendo, os resultados da coleta de dados são apresentados na Tabela 8,9 . 
Tabela 8. Resultado da aplicação das métricas - quinto período

\begin{tabular}{|c|c|c|c|c|c|c|}
\hline Métrica & Descrição & Insatisfeito & $\begin{array}{c}\text { Pouco } \\
\text { Satisfeito }\end{array}$ & Satifeito & Médio & \begin{tabular}{c|c} 
Muito \\
Satisfeiro
\end{tabular} \\
\hline \multicolumn{7}{|c|}{ Questão 1.1: Qual o grau de compreensão da linguagem utilizada pela documentação do framework? } \\
\hline M1.1.1) & $\begin{array}{l}\text { Grau de facilidade para compreender e completar as tarefas de forma rápida e eficiente com } \\
\text { o auxilio da documentação? }\end{array}$ & $43 \%$ & $29 \%$ & $14 \%$ & $14 \%$ & \\
\hline M1.1.2) & Grau de adequação do conteúdo da documentação ? & $0 \%$ & $43 \%$ & $43 \%$ & $14 \%$ & $0 \%$ \\
\hline \multicolumn{7}{|c|}{ Questão 1.2: Qual o grau de compreensão das ilustraç̃oes e exemplos utilizados na documentaç̃a? } \\
\hline M1.2.1) & $\begin{array}{l}\text { Grau de facilidade para compreender as ilustrações e exemplos utilizados na documentação } \\
\text { e completar as tarefas de forma rápida e eficiente? }\end{array}$ & $43 \%$ & $43 \%$ & $14 \%$ & $0 \%$ & $0 \%$ \\
\hline M1.2.2) & Grau de adequação das ilustrações e exemplos utilizados no conteúdo da documentação ? & $29 \%$ & $43 \%$ & $29 \%$ & $0 \%$ & $0 \%$ \\
\hline \multicolumn{7}{|c|}{ Questão 2.1: Qual o grau do nivel de detalhe da documentação de utilização do framework? } \\
\hline M2.1.1) & Grau de satisfação com a apresentação da documentação ? & $43 \%$ & $29 \%$ & $29 \%$ & $0 \%$ & $0 \%$ \\
\hline M2.1.2) & Grau de integração entre os assuntos da documentação ? & $0 \%$ & $29 \%$ & $29 \%$ & $14 \%$ & $29 \%$ \\
\hline M2.1.3) & Grau de clareza da documentação ? & $29 \%$ & $43 \%$ & $29 \%$ & $0 \%$ & $0 \%$ \\
\hline \multicolumn{7}{|c|}{ Questão 2.2: Qual o grau de satisfação do usuário após utilizarem a doc } \\
\hline M2.2.1) & Grau do nivel de satisfação da leitura e simplicidade da documentação? & $29 \%$ & $29 \%$ & $43 \%$ & $0 \%$ & $0 \%$ \\
\hline M2.2.2) & Grau de consistência da documentação & $0 \%$ & $43 \%$ & $43 \%$ & $14 \%$ & $0 \%$ \\
\hline M2.2.3) & Grau de facilidade de manipulação da documentação & $0 \%$ & $29 \%$ & $29 \%$ & $43 \%$ & $0 \%$ \\
\hline
\end{tabular}

Tabela 9. Resultado da aplicação das métricas - nono período

\begin{tabular}{|c|c|c|c|c|c|c|}
\hline Métrica & Descrição & Insatisfeito & \begin{tabular}{|c|c|} 
Pouco \\
Satisfeito
\end{tabular} & Satifeito & Médio & $\begin{array}{c}\text { Muito } \\
\text { Satisfeiro }\end{array}$ \\
\hline \multicolumn{7}{|c|}{ Questão 1.1: Qual o grau de compreensão da linguagem utilizada pela documentação do framework? } \\
\hline M1.1.1) & $\begin{array}{l}\text { Grau de facilidade para compreender e completar as tarefas de forma rápida e eficiente com } \\
\text { o auxilio da documentação? }\end{array}$ & $0 \%$ & $0 \%$ & $29 \%$ & $57 \%$ & $14 \%$ \\
\hline M1.1.2) & Grau de adequação do conteúdo da documentação ? & $0 \%$ & $14 \%$ & $29 \%$ & $29 \%$ & $29 \%$ \\
\hline \multicolumn{7}{|c|}{ Questão 1.2: Qual o grau de compreensão das ilustraç̃es e exemplos utilizados na documentação? } \\
\hline M1.2.1) & $\begin{array}{l}\text { Grau de facilidade para compreender as ilustrações e exemplos utilizados na documentação } \\
\text { e completar as tarefas de forma rápida e eficiente? }\end{array}$ & $0 \%$ & $14 \%$ & $43 \%$ & $14 \%$ & $29 \%$ \\
\hline M1.2.2) & Grau de adequação das ilustrações e exemplos utilizados no conteúdo da documentação ? & $0 \%$ & $14 \%$ & $29 \%$ & $43 \%$ & $14 \%$ \\
\hline \multicolumn{7}{|c|}{ Questão 2.1: Qual o grau do nivel de detalhe da documentação de utilização do framework? } \\
\hline M2.1.1) & Grau de satisfação com a apresentação da documentação? & $0 \%$ & $0 \%$ & $0 \%$ & $43 \%$ & $57 \%$ \\
\hline M2.1.2) & Grau de integração entre os assuntos da documentação ? & $0 \%$ & $14 \%$ & $43 \%$ & $14 \%$ & $29 \%$ \\
\hline M2.1.3) & Grau de clareza da documentação? & $0 \%$ & $43 \%$ & $43 \%$ & $14 \%$ & $0 \%$ \\
\hline \multicolumn{7}{|c|}{ Questão 2.2: Qual o grau de satisfação do usuário após utilizarem a documentação d } \\
\hline M2.2.1) & Grau do nivel de satisfação da leitura e simplicidade da documentação ? & $0 \%$ & $0 \%$ & $43 \%$ & $43 \%$ & $14 \%$ \\
\hline M2.2.2) & Grau de consistência da documentação & $0 \%$ & $0 \%$ & $14 \%$ & $71 \%$ & $14 \%$ \\
\hline M2.2.3) & Grau de facilidade de manipulação da documentação & $0 \%$ & $0 \%$ & $0 \%$ & $71 \%$ & $29 \%$ \\
\hline
\end{tabular}

Para avaliar as documentações de utilização de framework foi utilizada a abordagem GQM que definiu os objetivos e questões que respondessem sobre o grau de compreensão da linguagem utilizada pela documentação, as ilustrações e exemplos, o nível de detalhe e a satisfação do usuário após utilizar a documentação oferecida, avaliando assim os resultados das opiniões sobre a facilidade de entendimento da documentação e sua usabilidade. Conforme definido pela abordagem GQM, através dos dados obtidos pelas métricas, constatou-se um alto grau de insatisfação na compreensão da linguagem utilizada pela documentação do framework (Questão 1.1) da estrutura de documentação definida pelos autores (Tabela 1) em relação à estrutura sugerida pela GCF (Figura 2). E como destaque desse item, é possível citar que a facilidade para compreender e completar as tarefas de forma rápida e eficiente com o GCF obteve uma avaliação bastante significativa, 57\% dos entrevistados responderam que estavam bastante satisfeitos. Em relação ao grau de compreensão das ilustrações e exemplos utilizados na documentação (Questão 1.2) os valores apresentam um elevado grau de satisfação dos alunos com a estrutura do GCF em relação à estrutura definida por eles próprios. Já o nível de satisfação ficou elevado com a apresentação da documentação, a integração entre os assuntos e a clareza dos itens no GCF comparados à estrutura de documentação definida pelos alunos no que se referente ao grau do nível de detalhe (Questão 2.1). Entretanto, o valor da métrica em relação à facilidade de manipulação entre as estrutura deixou evidente que a definição de uma estrutura padronizada, como a do GCF, pode trazer uma satisfação maior quanto à questão de reuso do framework (Questão 2.2). Dos resultados apresentados (Tabelas 8 e 9) em relação aos objetivos de avaliar a facilidade de entendimento da documentação de utilização de framework [Ramos 2004] e a usabilidade do mesmo [Usability 2008] o GCF demonstrou ser mais eficaz oferecendo resultados mais expressivos em relação a utilizada pelos alunos. 


\section{Conclusão}

A medição da qualidade dos documentos de utilização de framework é uma estratégia importante para ajudar a otimizar a curva de aprendizagem e aumentar o reúso dos frameworks. Neste artigo foi apresentada uma proposta para avaliar documentos elaborados pelos alunos do $5^{\circ}$ ano do curso de engenharia em relação GCF baseado no modelo GQM. Os resultados aqui apresentados demonstraram uma variação no grau de satisfação acentuada entre os alunos, principalmente em relação à estrutura sugerida pelo GCF. Como lições aprendidas foram constatadas que: a) não basta apenas a definição de uma estrutura padronizada, mas que a mesma esteja associada a conteúdos melhores elaborados pelos usuários; b) O GQM mostrou ser um método simples para avaliar e oferecer maior qualidade em relação à documentação de utilização de framework. Para as próximas atividades de avaliação, a abordagem será aplicada em um ambiente real de desenvolvimento não controlado e serão estabelecidos novos objetivos e metas de verificação buscando se aproximar de um valor ideal definido pelos usuários.

\section{Referências}

Basili, V. e Rombach, H. (1994) "Goal question metric paradigm", Encyclopedia of software engineering.

Booch, G. Designing an Application Framework. (1994) Dr. Dobb’s Journal 19.

Fenton, N. E, Pfleeger, L., (1994) "Software metrics a rigorous and practical approach", PWS Publishing Company.

Gomes, A., Oliveira, K. M., Rocha, A. R. (2001) Métricas para Medição e Melhoria de Processo de Software. Rio de Janeiro: COPPE/UFRJ.

ISO 9126 (2001) International Organization for Standardization - "Software engineering - Product quality - Part 1".

Johnson, R. E., (1992) Documenting frameworks using patterns., SIGPLAN Notices, New York, v.27, n.10.

Krasner, G.E., Pope, S.T., (1988) A Cookbook for Using the Model-View-Controller User Interface Paradigm in Smalltalk-80, Journal of Object-Oriented Programming.

Pree, W., (1995) Design Patterns for Object-Oriented Software Development, AddisonWesley Publishing Company.

Ramos, C. S.; Oliveira, K. M (2004). Conhecendo Sistemas Legados através de Métricas de Software. In: III Simpósio Brasileiro de Qualidade de Software.

SAGP, (2008) Software Acquisition Gold Practice - Goal-Question-Metric (GQM) Approach, https://www.goldpractices.com/practices/gqm/.

Souza, F M, (2008) GCF - Uma Abordagem para o Desenvolvimento de uma Guia de Colaboração de Framework. São José dos Campos.

Soligen, R. e Berghout, E., (1999) "The goal/question/metric method - A pratical guide for quality improvement of software development".

Usability. Your Guide for Development Usable \& Useful Web Sites (2008). Disponível em: <http://usability.gov> 